\title{
ON CERTAIN CLASSES OF ANALYTIC FUNCTIONS OF COMPLEX ORDER DEFINED BY DZIOK-SRIVASTAVA OPERATOR
}

\author{
G. MurugusundaramoORTHY, T. ROSY AND S. SiVASUBRAMANIAN
}

Abstract. Making use of the generalized hypergeometric functions, we introduced certain new subclasses of analytic functions defined by Dziok-Srivastava operator in the unit disc. The main object of the present paper is to investigate the various properties and characteristics of analytic functions belonging to the subclasses $S_{n}(l, m, \lambda, b, \gamma)$ satisfying the inequality

$$
\left|\frac{1}{b}\left(\frac{z\left(H_{m}^{l}\left[\alpha_{1}, \beta_{1}\right] f(z)\right)^{\prime}}{(1-\lambda) H_{m}^{l}\left[\alpha_{1}, \beta_{1}\right] f(z)+\lambda z\left(H_{m}^{l}\left[\alpha_{1}, \beta_{1}\right] f(z)\right)^{\prime}}-1\right)\right|<\gamma,
$$

where $z \in U, b \in \mathbb{C} \backslash\{0\}, 0<\gamma \leqslant 1,0 \leqslant \lambda \leqslant 1$ and $H_{m}^{l}\left[\alpha_{1}, \beta_{1}\right] f(z)$ is Dziok-Srivastava operator. Also let $R_{n}(l, m, \lambda, b, \gamma)$ be an another subclass satisfying the inequality

$$
\left|\frac{1}{b}\left((1-\lambda) \frac{H_{m}^{l}\left[\alpha_{1}, \beta_{1}\right] f(z)}{z}+\lambda\left(H_{m}^{l}\left[\alpha_{1}, \beta_{1}\right] f(z)\right)^{\prime}-1\right)\right|<\gamma
$$

where $z \in U, b \in \mathbb{C} \backslash\{0\}, 0<\gamma \leqslant 1,0 \leqslant \lambda \leqslant 1$ and $H_{m}^{l}\left[\alpha_{1}, \beta_{1}\right] f(z)$ is given by by Dziok-Srivastava [7]. Apart from deriving a set of coefficient bounds for each of these function classes, we establish several inclusion relationships involving the $(n, \delta)$-negihborhoods of analytic functions with negative coefficients belonging to these subclasses.

Mathematics subject classification (2000): 30C45.

Key words and phrases: Univalent, starlike, convex, $(n, \delta)$-neighborhood, inclusion relations, Hadamard product, generalized hypergeometric functions.

\section{REFERENCES}

[1] O. Altintas, On a subclass of certain starlike functions with negative coefficients, Math. Japon., 36(3) (1991), 489-495.

[2] O. AltinTAS AND S. OwA, Neighborhood of certain analytic functions with negative coefficients, Internat. J. Math. Math. Sci., 19 (1996), 797-800.

[3] O. Altintas, O. OZKa AND H.M. SRIVASTAVA, Neighborhoods of a class analytic functions with negative coefficients, Appl. Math. Letters., 13 (2000), 63-67.

[4] O. AltinTAS, O. OZKA AND H.M. SRIVASTAVA, Neighborhoods of a certain family of multivalent functions with negative coefficients, Comput. Math. Appl., 47 (2004), 1667-1672.

[5] B.C. CARLSON, Special Functions of Applied Mathematics, Academic Press, New York 1977.

[6] B.C. CARLSON AND S.B. SHAFFER, Starlike and prestarlike hypergeometric functions, SIAM, J. Math. Anal., 15 (2002), 737-745.

[7] J. DZIOK AND H.M. SRIVASTAVA, Certain subclasses of analytic functions associated with the generalized hypergeometric function, Intergral Transform and Spec. Funct., 14 (2003), 7-18.

[8] A.W. Goodman, Univalent functions and nonanalytic curves, Proc. Amer. Math. Soc., 8 (1957), 598-601. 
[9] H.O. GÜNEY AND S. EKER, Neighborhoods of a class analytic functions with negative coefficients, Inter. Math. Forum, 1(9) (2006), 429-432.

[10] G. MurugusundaramoorThy AND H.M. SRIVASTAVA, Neighborhoods of certain classes of analytic functions of complex order, J. Inequal. Pure Appl. Math., 5 (2) (2004), Art. 24.8 pp.

[11] M.A. NASR AND M.K. AOUF, Starlike function of complex order, J. Natur. Sci. Math., 25 (1985), 1-=12.

[12] S. OWA, On the distortion theorems - I, Kyungpook. Math. J., 18 (1978), 53-59.

[13] S. PonNUSAMY AND S. SABAPATHY, Geometric properties of generalized hypergeometric functions, Ramanujan Journal, 1 (1997), 187-210.

[14] E.D. RainVILle, Special Functions, Chelsea Publishing Company, New York 1960.

[15] St. Ruscheweyh, New criteria for univalent functions, Proc. Amer. Math. Soc., 49 (1975), 109-115.

[16] S. RuCHEWEYH, Neighborhoods of univalent functions, Proc. Amer. Math. Soc., 81 (1981), 521-527.

[17] H. Silverman, Univalent functions with negative coefficients, Proc. Amer. Math. Soc., 51 (1975), $109-116$.

[18] H. SiLVERMAN, Neighborhoods of a classes of analytic function, Far. East. J. Math. Sci., 3(2) (1995), $165-169$.

[19] H.M. SRIVASTAVA AND S. OWA, Some characterization and distortion theorems involving fractional calculus, generalized hypergeometric functions, Hadamard products, linear operators and certain subclasses of analytic functions, Nagoya Math. J., 106 (1987), 1-28.

[20] P. WIATROWSKI, On the coefficients of some family of holomorphic functions, Zesyty Nauk. Uniw. Lodz Nauk. Mat- Przyord., 39(2) (1970), 75-85. 